\title{
Correction to: Elucidating the distinct interactive impact of cadmium and nickel on growth, photosynthesis, metal-homeostasis, and yield responses of mung bean (Vigna radiata L.) varieties
}

\author{
Muhammad Aqeel $^{1} \cdot$ Noreen Khalid $^{2} \cdot$ Aasma Tufail $^{3} \cdot$ Rana Zaheer Ahmad $^{4} \cdot$ Muhammad Salim Akhter $^{5}$. \\ Muhammad Luqman ${ }^{6}$. Muhammad Tariq Javed ${ }^{7} \cdot$ Muhammad Kashif Irshad $^{8} \cdot$ Saad Alamri $^{9,10}$. \\ Mohamed Hashem $^{9,11}$. Ali Noman ${ }^{7}$ \\ Published online: 23 February 2021 \\ (C) Springer-Verlag GmbH Germany, part of Springer Nature 2021
}

\section{Correction to: Environ Sci Pollut Res}

https://doi.org/10.1007/s11356-021-12579-5

The authors provide this corrigendum to correct affiliation of authors Muhammad Tariq Javed ${ }^{7}$, Ali Noman ${ }^{7}$ and Muhammad Kashif Irshad ${ }^{8}$ which were mistakenly interchanged in the original article. The correct affiliation of authors is presented in this paper. The authors would like to apologise for any inconvenience caused.

Please note that this correction is not related to the contents of the research article, the analysis of the data, or the presentation of the results, does not affect in any way the conclusions of this article.

The online version of the original article can be found at https://doi.org/ $10.1007 / \mathrm{s} 11356-021-12579-5$

Ali Noman

alinoman@gcuf.edu.pk

1 State Key Laboratory of Grassland Agro-ecosystems, School of Life Science, Lanzhou University, Lanzhou, Gansu 730000, People's Republic of China

2 Department of Botany, Government College Women University Sialkot, Sialkot, Pakistan

3 Department of Botany, Division of Science and Technology, University of Education, Lahore, Pakistan

4 College of Pastoral Agriculture Science and Technology, Lanzhou University, Lanzhou 730000, People's Republic of China

5 Institute of Pure and Applied Biology, Bahauddin Zakariya University, Multan, Pakistan
Department of Botany, University of Agriculture Faisalabad, Faisalabad, Pakistan

7 Department of Botany, Government College University Faisalabad, Faisalabad, Pakistan

8 Department of Environmental Science, Government College University Faisalabad, Faisalabad, Pakistan

9 Department of Biology, College of Science, King Khalid University, Abha 61413, Saudi Arabia

10 Prince Sultan Ben Abdulaziz Center for Environmental and Tourism Research and Studies, King Khalid University, Abha, Saudi Arabia

11 Botany and Microbiology Department, Faculty of Science, Assiut University, Assiut 71516, Egypt 